\title{
THE MICRO-SUPPORT OF THE COMPLEX DEFINED BY A CONVOLUTION OPERATOR IN TUBE DOMAINS
}

\author{
RYUICHI ISHIMURA and YASUNORI OKADA \\ Department of Mathematics and Informatics, Faculty of Sciences, Chiba University \\ 1-33 Yayoi-cho, Inage-ku, Chiba, 263 Japan \\ E-mail: ishimura@math01.c.chiba-u.ac.jp
}

0. Introduction. In the present report, we consider the convolution operator $u *$ defined by a hyperfunction $u(x)$ with compact support, operating on holomorphic functions in tube domains invariant by any real translation. In all what follows we impose the natural condition called (S) introduced by Kawai [Kaw]. This condition is in fact equivalent to the classical property of completely regular growth in pure-imaginary directions $([\mathrm{IOk}])$. First we have under this condition (S), the existence of solutions in any open tube domain. And conversely, we also have that the existence of solutions in some special tube domain implies the condition (S), meaning that (S) is a sufficient and almost necessary condition for the existence. Secondly, we will define the characteristic set $\operatorname{Car}(u *)$ of $u *$ and prove that all solutions of the homogeneous equation $u * f=0$ are extended holomorphically to any non-characteristic direction. Finally, by these results, we will conclude under the condition (S) that the micro-support of the complex defined by the operator $u *$ is included in the characteristic set $\operatorname{Car}(u *)$. Most of the results are based on our paper $[\mathrm{IO}]$ and on $[\mathrm{IOk}]$ and we refer to them for the details of proofs.

The authors take this occasion to express their thanks to Polish Academy of Sciences and Institute of Mathematics for inviting one of the authors and for all hospitality during his stay in Warszawa, especially to Professor B. Ziemian and also to secretaries of Banach Center.

1. Problem and notations. Let $\tau$ be the projection $\mathbb{R}^{n} \times \sqrt{-1} \mathbb{R}^{n} \rightarrow \sqrt{-1} \mathbb{R}^{n}$ and set

$$
\mathcal{O}^{\tau}=R \tau_{*} \mathcal{O}_{\mathbb{C}^{n}}
$$

1991 Mathematics Subject Classification: 35R50, 46F15.

The paper is in final form and no version of it will be published elsewhere. 
where we denote by $\mathcal{O}_{\mathbb{C}^{n}}$ the sheaf of holomorphic functions on $\mathbb{C}^{n}$. It is, in fact, a sheaf on $\sqrt{-1} \mathbb{R}^{n}$. Let $u(x)$ be a hyperfunction with compact support $M$ on $\mathbb{R}^{n}$ and consider the complex

$$
\mathcal{S}: 0 \rightarrow \mathcal{O}^{\tau} \stackrel{u *}{\longrightarrow} \mathcal{O}^{\tau} \rightarrow 0 .
$$

Then to consider the existence and the analytic continuation at the same time, we formulate (cf. Kashiwara-Schapira [KS]):

Problem 1.1. Define the "characteristic set" Car $(u *)$ to be a natural generalization of the classical case of partial differential operators and estimate the micro-support $\operatorname{SS}(\mathcal{S})$ by $\operatorname{Car}(u *)$.

For an analytic functional $T \in \mathcal{O}\left(\mathbb{C}^{n}\right)^{\prime}$, let $\hat{T}(\zeta)$ denote its Fourier-Borel transform:

$$
\hat{T}(\zeta)=\left\langle T, e^{z \cdot \zeta}\right\rangle_{z}
$$

In particular, if $u$ is a hyperfunction with compact support, its Fourier-Borel transform $\hat{u}(\zeta)$ is an entire function with infra-exponential growth on $\sqrt{-1} \mathbb{R}^{n}$.

\section{The condition (S)}

Definition 2.1 ([IOk]). We say that a subharmonic function $s(\xi)$ on $\mathbb{R}^{m}$ satisfies the condition (S) in the direction $\xi_{0}$ or simply it satisfies the condition $(S)_{\xi_{0}}$ if we have

For every small $\varepsilon>0$, there exists $N>0$ such that for any $r>N$, we have $\xi \in \mathbb{R}^{m}$ satisfying $\left|\xi-\xi_{0}\right|<\varepsilon$ and

$$
s(r \xi) / r^{\rho(r)} \geq \hat{h}_{s}^{*}\left(\xi_{0}\right)-\varepsilon
$$

where $h_{s}^{*}\left(\xi_{0}\right)$ is the regularized growth indicator of $s(\xi)$ (see for example Lelong-Gruman $[\mathrm{LG}])$. We also say that a holomorphic function $f(\zeta)$ defined in a part of a cone, outside of a bounded set, satisfies the condition (S) in the direction $\zeta_{0}$ or satisfies the condition $(S)_{\zeta_{0}}$ if, identifying $\mathbb{C}^{n}$ with $\mathbb{R}^{2 n}$, the subharmonic function $\ln |f(\zeta)|$ satisfies the above condition (S).

Remark 2.3. In [IOk], R. Ishimura and J. Okada proved that the condition (S) is nothing but the notion of regular growth, classical in the case of one variable, and considered by Lelong-Gruman [LG], for example, in the general case.

Remark 2.4. In the case of a hyperfunction with compact support $u(x)$, because its Fourier transform is of minimal type in real directions, that is, we have $h_{\hat{u}}^{*}(\xi)=0$ for every $\xi \in \mathbb{R}^{n}$, definition 2.1 is a natural generalization of the definition of Kawai [Kaw].

In the sequel, we denote for $R>0$ and $\zeta_{0} \in \mathbb{C}^{n}$ by $B\left(\zeta_{0}, R\right)$ the open ball in $\mathbb{C}^{n}$ of centre $\zeta_{0}$ and with radius $R$.

Throughout the paper, we will suppose the condition (S) for the entire function $f(\zeta)=$ $\hat{u}(\zeta)$ in pure imaginary directions:

For every $\varepsilon>0$, there exists $N>0$ such that for any $\sqrt{-1} \eta \in \sqrt{-1} \mathbb{R}^{n}$ with $|\eta|>N$, we can find $\zeta \in \mathbb{C}^{n}$ which satisfies

$$
|\sqrt{-1} \eta-\zeta|<\varepsilon|\eta|, \quad|f(\zeta)| \geq e^{-\varepsilon|\eta|} .
$$

First we have the following division lemma under the condition (S). 
LEMmA 2.6. Let $f, g$ and $h \in \mathcal{O}\left(\mathbb{C}^{n}\right)$ be entire functions of exponential type and $M$ and $K$ be two compact sets in $\mathbb{C}^{n}$. Suppose that $f g=h$ and that for every $\varepsilon>0$, there exist $A_{\varepsilon}>0$ and $B_{\varepsilon}>0$ such that for any $\zeta \in \mathbb{C}^{n}$, we have

$$
\begin{aligned}
& \ln |f(\zeta)| \leq A_{\varepsilon}+H_{M}(\zeta)+\varepsilon|\zeta|, \\
& \ln |h(\zeta)| \leq B_{\varepsilon}+H_{K}(\zeta)+\varepsilon|\zeta| .
\end{aligned}
$$

Assume also that $f$ satisfies the condition $(S)$. Then for any a $>0$ there exists a compact set $L \subset \mathbb{C}^{n}$ satisfying $\tau(L) \subset \tau(K+B(0 ; a))$ and for every $\varepsilon>0$, there exists $C_{\varepsilon}>0$ such that we have for any $\zeta \in \mathbb{C}^{n}$,

$$
\ln |g(\zeta)| \leq C_{\varepsilon}+H_{L}(\zeta)+\varepsilon|\zeta| .
$$

Sketch of proof. For any $\varepsilon>0$ with $0<\varepsilon<\frac{1}{2}$, let $\Gamma_{\varepsilon}$ be a conic $\varepsilon$-neighborhood of $\sqrt{-1} \mathbb{R}^{n}$ i.e. $\Gamma_{\varepsilon}:=\left\{\zeta \in \mathbb{C}^{n} \mid\right.$ there exists $\sqrt{-1} \eta \in \sqrt{-1} \mathbb{R}^{n}$ such that $\left.|\zeta-\sqrt{-1} \eta|<\varepsilon|\eta|\right\}$. First we prove the estimate (2.9) for $\zeta \in \Gamma_{\varepsilon}$ : By the condition (S), there exists $N>0$ so that if $|\zeta|>N$, we can find $\zeta^{\prime} \in \mathbb{C}^{n}$ satisfying $\left|\zeta^{\prime}-\sqrt{-1} \eta\right|<\varepsilon|\eta|$ and $\left|f\left(\zeta^{\prime}\right)\right| \geq e^{-\varepsilon|\eta|}$.

We recall a lemma of Harnack-Malgrange-Hörmander ([H1], Lemma 3.1):

LEMMA 2.10. If $F(\zeta), H(\zeta)$ and $G(\zeta)=H(\zeta) / F(\zeta)$ are three holomorphic functions in the open ball $B(0 ; R)$, and if $|F(\zeta)|<A,|H(\zeta)|<B$ on $B(0 ; R)$, then

$$
|G(\zeta)| \leq B A^{\frac{2|\zeta|}{R-|\zeta|}}|F(0)|^{-\frac{R+|\zeta|}{R-|\zeta|}}
$$

for all $\zeta \in B(0 ; R)$.

We apply this lemma for the ball $B\left(\zeta^{\prime} ; 3 \varepsilon|\eta|\right)$. By $(2.8)$, setting $k:=\sup _{z \in K}|z|$, for any $\delta>0$ with $\delta \leq \min (\varepsilon, 1)$, we have

$$
\sup _{\zeta^{\prime \prime} \in B\left(\zeta^{\prime} ; 3 \varepsilon|\varepsilon|\right)} \ln \left|h\left(\zeta^{\prime \prime}\right)\right| \leq B_{\varepsilon}+4(k+1) \varepsilon|\eta|+H_{K}(\sqrt{-1} \eta) .
$$

In the same way, by setting $m:=\sup _{x \in M}|x|$ and noting that $M \subset \mathbb{R}^{n}$, using (2.7) we have

$$
\sup _{\zeta^{\prime \prime} \in B\left(\zeta^{\prime} ; 3 \varepsilon|\varepsilon|\right)} \ln \left|f\left(\zeta^{\prime \prime}\right)\right| \leq A_{\varepsilon}+4(m+1) \varepsilon|\eta| .
$$

So remarking that $\zeta \in B\left(\zeta^{\prime} ; 2 \varepsilon|\eta|\right)\left(\subset B\left(\zeta^{\prime} ; 3 \varepsilon|\eta|\right)\right)$, by lemma 2.10, we have

$$
\begin{aligned}
\ln |g(\zeta)| \leq & B_{\varepsilon}+4(k+1) \varepsilon|\eta|+H_{K}(\sqrt{-1} \eta) \\
& +\frac{2\left|\zeta-\zeta^{\prime}\right|}{3 \varepsilon|\eta|-\left|\zeta-\zeta^{\prime}\right|}\left(A_{\varepsilon}+4(k+1)\right) \varepsilon|\eta|+\frac{3 \varepsilon|\eta|+\left|\zeta-\zeta^{\prime}\right|}{3 \varepsilon|\eta|-\left|\zeta-\zeta^{\prime}\right|} \varepsilon|\eta| \\
\leq & B_{\varepsilon}+4 A_{\varepsilon}+H_{K}(\sqrt{-1} \eta)+[4(k+4 m)+25] \varepsilon|\eta| .
\end{aligned}
$$

So for any $\varepsilon>0$, we can find a constant $C_{\varepsilon}^{\prime}>0$ such that

$$
\ln |g(\zeta)| \leq C_{\varepsilon}^{\prime}+H_{K}(\sqrt{-1} \eta)+\varepsilon|\eta|
$$

for $\zeta \in \Gamma_{\varepsilon}$. Next we consider the estimate (2.10) for all $\zeta \in \mathbb{C}^{n}$. We remark that in the case $n=1$, by the Phragmén-Lindelöf principle and the estimate in $\Gamma_{\varepsilon}$, we have the desired estimate, and in the general case when $n \geq 1$, we can find directly a constant $C_{\varepsilon}>0$ so that the estimate (2.10) follows. 
3. Vanishing of the first cohomology group. Under the condition (S), by the standard method as in Malgrange [M], we have the surjectivity:

THEOREM 3.1. Let $u(x)$ be a hyperfunction with compact support. Assume that $\hat{u}(\zeta)$ satisfies the condition $(\mathrm{S})$. Then for any open set $\sqrt{-1} \omega \subset \sqrt{-1} \mathbb{R}^{n}$, the operator

$$
u *: \mathcal{O}^{\tau}(\sqrt{-1} \omega) \rightarrow \mathcal{O}^{\tau}(\sqrt{-1} \omega)
$$

is surjective.

Corollary 3.2. We have $\operatorname{SS}\left(H^{1}(\mathcal{S})\right)=0$.

Conversely, by means of Favorov [F] and Morzhakov [Mo2] (see also Lelong-Gruman $[\mathrm{LG}]$ ), we also note that the condition $(\mathrm{S})$ is a necessary condition for the surjectivity in the following sense:

THEOREM 3.3. Let $\sqrt{-1} \omega$ is a strictly convex bounded open domain with $C^{2}$-boundary in $\sqrt{-1} \mathbb{R}^{n}$. Then if $u *: \mathcal{O}^{\tau}(\sqrt{-1} \omega) \rightarrow \mathcal{O}^{\tau}(\sqrt{-1} \omega)$ is surjective, then $f(\zeta):=\hat{u}(\zeta)$ has to satisfy the condition $(\mathrm{S})$.

Remark 3.4. In the case of $n=1$, by theorem 3.1 and by the proof of Theorem 7 of Morzhakov [M2] (or of Lelong-Gruman [LG], Theorem 9.35), we can conclude that the condition ( $\mathrm{S}$ ) is nothing but the property of (completely) regular growth (see e.g. Levin $[\mathrm{L}])$ in the pure imaginary directions $\sqrt{-1} \mathbb{R}^{n}$. But in the general case of $n \geq 1$, we can also prove the equivalence of $(\mathrm{S})$ and the property of regular growth in Lelong-Gruman sense $[\mathrm{LG}]$ (see $[\mathrm{IOk}])$.

4. The characteristic set and the continuation of homogeneous solutions. For the 0-th cohomology group of the complex $\mathcal{S}$, under the condition $(\mathrm{S})$ for $u \in \mathcal{B}_{c}\left(\mathbb{R}^{n}\right)$, we shall now solve the prolongation problem of $\mathcal{O}^{\tau}$-solutions of the homogeneous equation $u * f=0$ by the method of Kiselman [Ki] and Sébbar [Sé]. We denote the sheaf of $\mathcal{O}^{\tau}$-solutions by $\mathcal{N}$, that is, for any open set $\sqrt{-1} \omega \subset \sqrt{-1} \mathbb{R}^{n}$, we set

$$
\mathcal{N}(\sqrt{-1} \omega):=\left\{f \in \mathcal{O}^{\tau}(\sqrt{-1} \omega) \mid u * f=0\right\}
$$

For an open set $\sqrt{-1} \Omega \subset \sqrt{-1} \mathbb{R}^{n}$ with $\sqrt{-1} \omega \subset \sqrt{-1} \Omega$, the problem is to get a condition for the restriction map $r: \mathcal{N}(\sqrt{-1} \Omega) \rightarrow \mathcal{N}(\sqrt{-1} \omega)$ to be surjective.

We note that the subspace $\mathcal{N}(\sqrt{-1} \omega)$ of the (FS) space $\mathcal{O}^{\tau}(\sqrt{-1} \omega)$, endowed with the induced topology, becomes a closed subspace. We have

Proposition 4.1. Let $\sqrt{-1} \omega$ and $\sqrt{-1} \Omega$ be two open subsets of $\sqrt{-1} \mathbb{R}^{n}$ with $\sqrt{-1} \omega \subset$ $\sqrt{-1} \Omega$. Assume that $u$ satisfies $(\mathrm{S})$. Then the image $\operatorname{Im} r$ of the restriction map $r$ is dense in $\mathcal{N}(\sqrt{-1} \omega)$.

Sketch of proof. Let $E$ be the space of exponential-polynomial solutions and $E^{\circ}$ its polar set in $\mathcal{O}\left(\mathbb{C}^{n}\right)^{\prime}$. We shall show that $E$ is dense in $\mathcal{N}(\sqrt{-1} \omega)$. To do this, it is sufficient, by Hahn-Banach's theorem, to prove that any functional $T \in E^{\circ} \cap \mathcal{O}\left(\mathbb{R}^{n} \times\right.$ $\sqrt{-1} \omega)^{\prime}$ is orthogonal to $\mathcal{N}(\sqrt{-1} \omega)$, and by the following lemma we have the desired result: 
Lemma 4.2 (B. Malgrange $[\mathrm{M}]) . T \in \mathcal{O}\left(\mathbb{C}^{n}\right)^{\prime}$ belongs to $E^{\circ}$ if and only if there exists an entire function $g(\zeta)$ satisfying

$$
\hat{T}(\zeta)=g(\zeta) \hat{u}(-\zeta)
$$

In order to present the continuation theorem, we will prepare the notion of characteristics which is a natural generalization of the case of usual differential operators of finite order with constant coefficients. We define the sphere at infinity $S_{\infty}^{2 n-1}$ by $\mathbb{C}^{n} / \mathbb{R}_{+}$ and consider the compactification with directions $\mathbb{D}^{2 n}=\mathbb{C}^{n} \sqcup S_{\infty}^{2 n-1}$ of $\mathbb{C}^{n}$. For any $\zeta \in \mathbb{C}^{n}, \zeta \neq 0$, we write $\zeta \infty \in S_{\infty}^{2 n-1}$ for the accumulating point of $\{t \zeta \mid t>0\}$ at $S_{\infty}^{2 n-1}$, i.e. $\{\zeta \infty\}=\left(\right.$ the closure of $\{t \zeta \mid t>0\}$ in $\left.\mathbb{D}^{2 n}\right) \cap S_{\infty}^{2 n-1}$. We denote by $\sqrt{-1} S_{\infty}^{n-1}$ the pure-imaginary sphere at infinity $\left\{(\xi+\sqrt{-1} \eta) \infty \in S_{\infty}^{2 n-1} \mid \xi=0\right\}$, which is a closed subset of $S_{\infty}^{2 n-1}$.

For a hyperfunction $u$ with compact support, using the modulus of the Fourier-Laplace transform $f=\hat{u}$ of $u$, we define the characteristics $\operatorname{Car}(u *)$ as follows: for positive $\varepsilon$ we set

$$
\begin{aligned}
& V_{f}(\varepsilon):=\left\{\zeta \in \mathbb{C}^{n}\left|e^{\varepsilon|\zeta|}\right| f(\zeta) \mid<1\right\}, \\
& W_{f}(\varepsilon):=S_{\infty}^{2 n-1} \cap\left(\text { the closure of } V_{f}(\varepsilon) \text { in } \mathbb{D}^{2 n}\right), \\
& W_{f}:=\text { the closure of } \bigcup_{\varepsilon>0} W_{f}(\varepsilon) .
\end{aligned}
$$

Now we define the characteristic set of $u *$.

DEFINITION 4.3. With the above notation, we put

$$
\operatorname{Car}(u *):=W_{f} \cap \sqrt{-1} S_{\infty}^{n-1} .
$$

We remark that in the case of differential operators of finite order with constant coefficients, the notion of the characteristic set coincides with the usual one defined as the accumulating directions of zeros of the principal symbol. We note that the direction $\sqrt{-1} \rho \infty \in \sqrt{-1} S_{\infty}^{n-1}$ does not belong to $\operatorname{Car}(u *)$ if and only if there exist a conic neighborhood $\Gamma \subset \mathbb{D}^{2 n}$ of $\sqrt{-1} \rho$, positive $N$ and an infra-linear function $\lambda: \mathbb{R}_{+} \rightarrow \mathbb{R}_{+}$such that $f(\zeta)$ satisfies the estimate

$$
|f(\zeta)|>e^{-\lambda(|\zeta|)}
$$

on $\Gamma \cap \mathbb{C}^{n} \cap\{|\zeta|>N\}$, where $\lambda(t)$ is said to be infra-linear if for any positive $\varepsilon$, we can find a constant $C$ satisfying

$$
\lambda(t) \leq \varepsilon t+C \quad \text { for any } t>0 .
$$

For this $\operatorname{Car}(u *)$ and an open convex $\sqrt{-1} \omega \subset \sqrt{-1} \mathbb{R}^{n}$, we now put $\sqrt{-1} \Omega:=$ the interior of

$$
\bigcap_{\sqrt{-1} \eta \infty \in \operatorname{Car}(u *)^{a}}\left\{\sqrt{-1} y \in \sqrt{-1} \mathbb{R}^{n} \mid\langle\sqrt{-1} y, \sqrt{-1} \eta\rangle \leq H_{\sqrt{-1} \omega}(\sqrt{-1} \eta)\right\},
$$

here ${ }^{a}$ means the antipodal. Then $\sqrt{-1} \Omega$ becomes an open convex subset in $\sqrt{-1} \mathbb{R}^{n}$ containing $\sqrt{-1} \omega$. Moreover for any compact $L$ in $\mathbb{R}^{n} \times \sqrt{-1} \Omega$, we can take compact $K$ in $\mathbb{R}^{n} \times \sqrt{-1} \omega$ such that

$$
H_{L}(\zeta) \leq H_{K}(\zeta) \quad \text { for any } \zeta \in \mathbb{C}^{n} \text { such that } \sqrt{-1} \eta \infty \in \operatorname{Car}(u *) \text {. }
$$


Under the above situation we state the most important theorem of this article.

THEOREM 4.4. Let $u$ be a hyperfunction with compact support, and let a pair of open sets $\sqrt{-1} \omega \subset \sqrt{-1} \Omega$ be as above. Assume that $\hat{u}$ satisfies the condition (S). Then the restriction $r_{\Omega}^{\omega}: \mathcal{N}(\sqrt{-1} \Omega) \rightarrow \mathcal{N}(\sqrt{-1} \omega)$ is surjective.

Proof. Remarking that the restriction $r_{\Omega}^{\omega}: \mathcal{N}(\sqrt{-1} \Omega) \rightarrow \mathcal{N}(\sqrt{-1} \omega)$ has a dense image, the transpose ${ }^{t} r_{\Omega}^{\omega}: \mathcal{N}(\sqrt{-1} \Omega)^{\prime} \leftarrow \mathcal{N}(\sqrt{-1} \omega)^{\prime}$ is injective and we need only show its surjectivity. By Hahn-Banach's theorem, any functional $T \in \mathcal{N}(\sqrt{-1} \Omega)^{\prime}$ has an extension $T_{1} \in \mathcal{O}\left(\mathbb{R}^{n} \times \sqrt{-1} \Omega\right)^{\prime}$, and let us choose $S \in \mathcal{O}\left(\mathbb{R}^{n} \times \sqrt{-1} \omega\right)^{\prime}$ so that $T_{1}$ and $S$ coincide on $\mathcal{N}(\sqrt{-1} \Omega)$. To do this, we use the following division lemma.

LEMMA 4.5. Let $L$ and $K$ be a pair of compact subsets of $\mathbb{C}^{n}$ satisfying the estimate (4.2) and $g(\zeta)$ be an entire function satisfying the estimate

$$
\ln |g(\zeta)| \leq H_{L}(\zeta)+C_{0}
$$

with a constant $C_{0}>0$. Then for any positive $\varepsilon$, there exists a constant $C_{\varepsilon}>0$ and entire functions $q(\zeta)$ and $r(\zeta)$ which satisfy

$$
\begin{aligned}
g(\zeta) & =\hat{u}(-\zeta) q(\zeta)+r(\zeta), \\
\ln |q(\zeta)| & \leq H_{L+\operatorname{supp} u}(\zeta)+\varepsilon|\zeta|+C_{\varepsilon}, \\
\ln |r(\zeta)| & \leq H_{K+\operatorname{supp} u}(\zeta)+\varepsilon|\zeta|+C_{\varepsilon} .
\end{aligned}
$$

Proof of the Lemma. For given positive $\varepsilon$, we divide $S_{\infty}^{2 n-1}$ into $\Lambda_{1} \cup \Lambda_{2}$ as

$$
\Lambda_{1}=\left\{\zeta \infty \in S_{\infty}^{2 n-1}\left|H_{L}(\zeta)<H_{K}(\zeta)+\frac{1}{4} \varepsilon\right| \zeta \mid\right\}, \quad \Lambda_{2}=S_{\infty}^{2 n-1} \backslash \Lambda_{1}
$$

Remark that $\Lambda_{1}$ is an open neighborhood of $\operatorname{Car}(u *)$. Since $\Lambda_{2}$ is compact, we can take an infra-linear function $\lambda(t)$ and positive $N$ such that

$$
\zeta \infty \in \Lambda_{2},|\zeta|>N \quad \text { implies } f(\zeta)>e^{-\lambda(|\zeta|)} .
$$

Moreover we can take this $\lambda$ so that it is $C^{\infty}$, equal to 1 in a neighborhood of $t=0$, and that $\left|\lambda^{\prime}(t)\right| \leq 1$.

According to the above, we divide $\mathbb{C}^{n}$ into the following three parts.

$$
\begin{aligned}
& \gamma_{1}=\left\{\zeta \in \mathbb{C}^{n}\left|\zeta \infty \in \Lambda_{1},\right| \zeta \mid>N\right\} \\
& \gamma_{2}=\left\{\zeta \in \mathbb{C}^{n}\left|\zeta \infty \in \Lambda_{2},\right| \zeta \mid>N\right\} \\
& \gamma_{3}=\left\{\zeta \in \mathbb{C}^{n}|| \zeta \mid \leq N\right\}
\end{aligned}
$$

Now we construct $q(\zeta)$ and $r(\zeta)$ as follows:

$$
\begin{aligned}
& q(\zeta):=\frac{g(\zeta)}{f(\zeta)}\left(1-\varphi\left(e^{\lambda(|\zeta|)} f(\zeta)\right)\right)+v(\zeta), \\
& r(\zeta):=g(\zeta) \varphi\left(e^{\lambda(|\zeta|)} f(\zeta)\right)-f(\zeta) v(\zeta),
\end{aligned}
$$

by choosing a suitable $C^{\infty}$ function $v(\zeta)$ on $\mathbb{C}^{n}$, where $\varphi(\tau)$ is a fixed $C^{\infty}$ function on $\mathbb{C}$ with

$$
0 \leq \varphi(\tau) \leq 1, \quad \varphi(\tau)= \begin{cases}1 & (|\tau| \leq 1 / 2) \\ 0 & (|\tau| \geq 1)\end{cases}
$$


The condition for $q$ and $r$ to be holomorphic is given as

$$
\bar{\partial} v=\frac{g(\zeta)}{f(\zeta)} e^{\lambda(|\zeta|)}\left\{\left(\frac{\partial \varphi}{\partial \tau} f(\zeta)+\frac{\partial \varphi}{\partial \bar{\tau}} \bar{f}(\zeta)\right) \frac{\lambda^{\prime}(|\zeta|)}{2|\zeta|} \zeta \cdot d \bar{\zeta}+\frac{\partial \varphi}{\partial \bar{\tau}} \bar{\partial} \bar{f}\right\} .
$$

We denote the right hand side by $w$ which satisfies $\bar{\partial} w=0$. Let us estimate $w$. In the outside of $\left\{1 / 2<e^{\lambda(|\zeta|)}|f(\zeta)|<1\right\}$, especially in $\gamma_{2}$, we have $w=0$ since $\varphi\left(e^{\lambda(|\zeta|)} f(\zeta)\right)$ is constant. In $\left\{1 / 2<e^{\lambda(|\zeta|)}|f(\zeta)|<1\right\}$, we have

$$
\begin{aligned}
|w| & \leq|g| e^{\lambda}(|\zeta|)\left\{\frac{1}{2}\left(\max _{|\tau| \leq 1} \frac{\partial \varphi}{\partial \tau}+\max _{|\tau| \leq 1} \frac{\partial \varphi}{\partial \bar{\tau}}\right)|d \bar{\zeta}|+\max _{|\tau| \leq 1} \frac{\partial \varphi}{\partial \bar{\tau}}\left|\frac{\bar{\partial} \bar{f}}{f}\right|\right\} \\
& \leq e^{H_{L}(\zeta)+\lambda(|\zeta|)} \max _{|\tau| \leq 1}\left(\frac{\partial \varphi}{\partial \tau}, \frac{\partial \varphi}{\partial \bar{\tau}}\right)\left(|d \bar{\zeta}|+2 e^{\lambda(|\zeta|)}|\partial f|\right)
\end{aligned}
$$

Since

$$
|\partial f| / \exp \left(H_{\operatorname{supp} u}(\zeta)+\varepsilon^{\prime}|\zeta|\right) \quad \text { is bounded for any } \varepsilon^{\prime}>0,
$$

we have, with some constant $C>0$,

$$
\ln |w| \leq H_{L}(\zeta)+H_{\text {supp } u}(\zeta)+2 \lambda(|\zeta|)+\frac{\varepsilon}{4}|\zeta|+C .
$$

Remarking that $H_{L}(\zeta)<H_{K}(\zeta)+\frac{\varepsilon}{4}|\zeta|$ holds in $\gamma_{1}$ and that $\gamma_{2}$ is compact, we get the estimate

$$
\ln |w| \leq H_{K}(\zeta)+H_{\operatorname{supp} u}(\zeta)+2 \lambda(|\zeta|)+\frac{\varepsilon}{2}|\zeta|+C^{\prime}
$$

in the whole $\mathbb{C}^{n}$, with another constant $C^{\prime}>0$.

By the same argument as in Sébbar [Sé] (pp. 205-206), using the estimate (4.1) for $\varepsilon / 4$, we conclude by Theorem 4.4.2 and the proof of Theorem 4.2.5 in Hörmander [H2] that there exists a solution $v \in C^{\infty}\left(\mathbb{C}^{n}\right)$ of (4.4) which satisfies the estimate

$$
\ln |v| \leq H_{K}(\zeta)+H_{\operatorname{supp} u}(\zeta)+\frac{3 \varepsilon}{4}|\zeta|+C^{\prime \prime}
$$

for some $C^{\prime \prime}>0$.

Finally let us estimate the growth of $q$ and $r$ constructed from this solution $v$. For $q$, we have

$$
|q-v|=|(1-\varphi) g / f| \begin{cases}=0 & \text { if }|f(\zeta)| \leq 1 / 2 \\ \leq 2 \exp \left(H_{K}(\zeta)+\lambda(|\zeta|)\right) & \text { if }|f(\zeta)|>1 / 2\end{cases}
$$

which shows the desired result. For $r$, we have

$$
\begin{aligned}
& |g \varphi| \leq \exp \left(H_{L}(\zeta)\right) \leq \exp \left(H_{K}(\zeta)+\frac{1}{4} \varepsilon|\zeta|\right) \quad \text { in } \gamma_{1}, \\
& |g \varphi|=0 \quad \text { in } \gamma_{2},
\end{aligned}
$$

which shows the desired result because of (4.3).

End of the proof of the Theorem. We can choose a compact $L \subset \mathbb{R}^{n} \times$ $\sqrt{-1} \Omega$ with $\hat{T}_{1}(\zeta) \leq$ Const $\cdot e^{H_{L}(\zeta)}$ and applying this lemma to $g=\hat{T}_{1}$, take functionals $Q \in \mathcal{O}\left(\mathbb{R}^{n} \times \sqrt{-1} \Omega\right)^{\prime}$ and $R \in \mathcal{O}\left(\mathbb{R}^{n} \times \sqrt{-1} \omega\right)^{\prime}$ which satisfy $\hat{T}_{1}=\hat{\tilde{u}} \cdot \hat{Q}+\hat{R}$. Then for any $g \in \mathcal{N}(\sqrt{-1} \Omega)$, we get

$$
\langle T, g\rangle=\left\langle T_{1}, g\right\rangle=\langle Q, u * f\rangle+\langle R, g\rangle=\langle R, g\rangle .
$$

This shows that $T={ }^{t} r_{\Omega}^{\omega} S$. 
5. Main theorem and examples. By the definition of micro-support (see [KS], Definition 5.1.2), Theorem 4.4 implies that

$$
\operatorname{SS}\left(H^{0}(\mathcal{S})\right) \subset \operatorname{Car}(u *) .
$$

So recalling Remark 5.1.4 in [KS], by (5.1) and Corollary 3.2, we resume our results of the preceding sections in the following final theorem:

THEOREN 5.1. Under the condition $(\mathrm{S})$, we have

$$
\mathrm{SS}(\mathcal{S}) \subset \operatorname{Car}(u *) \text {. }
$$

R e m a r k. For related topics, see the references; especially we cite a series of works of Korobernik [Ko1]-[Ko3], Napalkov [N], Morzhakov [MO1]-[Mo3], Epifanov [E1]-[E3], Tkachenko [T] and Berenstein, Gay, Méril, Struppa and Vidras ([BeGV], [BeSt1], [BeSt2], [MéSt] and [V]).

\section{References}

[Ao] T. Aoki, Existence and continuation of holomorphic solutions of differential equations of infinite order, Adv. in Math. 72 (1988), 261-283.

[BeGV] C. A. Berenstein, R. Gay and A. Vidras, Division theorems in the spaces of entire functions with growth conditions and their applications to PDE of infinite order, preprint.

[BeSt] C. A. Berenstein and D. C. Struppa, A remark on "convolutors in spaces of holomorphic functions", in: Lecture Notes in Math. 1276, Springer, 1987, 276-280.

[BeSt] - - -, Solutions of convolution equations in convex sets, Amer. J. Math. 109 (1987), $521-544$

[BoS] J. M. Bony et P. Schapira, Existence et prolongement des solutions holomorphes des équations aux dérivées partielles, Invent. Math. 17 (1972), 95-105.

[E1] O. V. Epifanov, Solvability of convolution equations in convex domains, Mat. Zametki 15 (1974), 787-796 (in Russian); English transl.: Math. Notes 15 (1974), $472-477$.

[E2] - On the surjectivity of convolution operators in complex domains, Mat. Zametki 16 (1974), 415-422. (in Russian); English transl.: Math. Notes 16 (1974), 837-841.

[E3] - Criteria for a convolution to be epimorphic in arbitrary regions of the complex plaine, Mat. Zametki 31 (1974), 695-705 (in Russian); English transl.: Math. Notes 31 (1982), 354-359.

[F] Yu. Favorov, On the addition on the indicators of entire and subharmonic functions of several variables, Mat. Sb. 105 (147) (1978), 128-140 (in Russian).

[H1] L. Hörmander, On the range of convoluton operators, Ann. of Math. 76 (1962), $148-170$.

[H2] - - An Introduction to Complex Analysis in Several Variables, Van Nostrand, 1966.

[I1] R. Ishimura, Théorèmes d'existence et d'approximation pour les équations aux dérivées partielles linéaires d'ordre infini, Publ. RIMS Kyoto Univ. 32 (1980), 393-415.

[I2] - Existence locale de solutions holomorphes pour les équations différentielles d'ordre infini, Ann. Inst. Fourier (Grenoble) 35 (3) (1985), 49-57. 
[I3] R. Ishimura, A remark on the characteristic set for convolution equations, Mem. Fac. Sci. Kyushu Univ. 46 (1992), 195-199.

[IO] R. Ishimura and Y. Okada, The existence and the continuation of holomorphic solutions for convolution equations in tube domains, Bull. Soc. Math. France 122 (1994), 413-433.

[IOk] - - - Sur la condition (S) de Kawai et la propriété de croissance régulière d'une fonction sous-harmonique et d'une fonction entière, Kyushu J. Math. 48 (1994), 257-263.

[Kan] A. Kaneko, Introduction to Hyperfunctions, Kluwer Acad. Publ., 1988.

[KS] M. Kashiwara and P. Schapira, Sheaves on Manifolds, Grundlehren Math. Wiss. 292, Springer, 1990.

[Kaw] T. Kawai, On the theory of Fourier hyperfunctions and its applications to partial differential equations with constant coefficients, J. Fac. Sci. Univ. Tokyo Sect. IA Math. 17 (1970), 467-517.

[Ki] C. O. Kiselman, Prolongement des solutions d'une équation aux dérivées partielles à coefficients constants, Bull. Soc. Math. France 97 (1969), 329-356.

[Ko1] Yu. F. Korobeŭnik, On solutions of some functional equations in classes of functions analytic in convex domains, Mat. Sb. 75 (1968), 225-234 (in Russian); English transl.: Math. USSR-Sb. 4 (1968), 203-211.

[Ko2] - , The existence of an analytic solution of an infinite order differential equation and the nature of its domain of analyticity, Mat. Sb. 80 (1969), 52-76 (in Russian); English transl.: Math. USSR-Sb. 9 (1969), 53-71.

[Ko3] - Convolution equations in the complex domain, Mat. Sb. 127 (1699 (1985) (in Russian); English transl.: Math. USSR-Sb. 55 (1985), 171-193.

[LG] P. Lelong and L. Gruman, Entire Functions of Several Complex Variables, Grundlehren Math. 282, Springer, 1986.

[L] B. Ja. Levin, Distribution of Zeros of Entire Functions, Trans. Math. Monographs 5, Amer. Math. Soc., 1964.

[M] B. Malgrange, Existence et approximation des solutions des équations aux dérivées partielles et des équations de convolution, Ann. Inst. Fourier (Grenoble) 6 (19551956), 271-354.

[MéSt] A. Méril and D. C. Struppa, Convolutors in spaces of holomorphic functions, in: Lecture Notes in Math. 1276, Springer, 1987, 253-275.

[Mo1] V. V. Morzhakov, Convolution equations in spaces of functions holomorphic in convex domains and on convex compacta in $\mathbb{C}^{n}$, Mat. Zametki 16 (1974), 431-440 (in Russian); English transl.: Math. Notes 16 (1974), 846-851.

[Mo2] - On epimorphicity of a convolution operator in a convex domains in $\mathbb{C}^{l}$, Mat. Sb. 132 (174) (1987), 352-370 (in Russian); Math. USSR-Sb. 60 (1988), 347-364.

[Mo3] - Convolution equations in convex domains of $\mathbb{C}^{n}$, in: Complex Anal. and Appl. '87, Sofia, 1989, 360-364.

[N] V. V. Napalkov, Convolution equations in multidimensional spaces, Mat. Zametki 25 (1979), 761-774 (in Russian); English transl.: Math. Notes 25 (1979).

[O] Y. Okada, Stability of convolution operators, Publ. RIMS Kyoto Univ., to appear.

[Sé] A. Sébbar, Prolongement des solutions holomorphes de certains opérateurs différentiels d'ordre infini à coefficients constants, in: Sém. Lelong-Skoda, Lecture Notes in Math. 822, Springer, 1980, 199-220. 
[T] V. A. Tkachenko, Equations of convolution type in spaces of analytic functionals, Izv. Akad. Nauk SSSR Ser. Mat. 41 (1977), 378-392 (in Russian); English transl.: Math. USSR-Izv. 11 (1977), 361-374.

[V] A. Vidras, Interpolation and division problems in spaces of entire functions with growth conditions and their applications, Doct. Diss., Univ. of Maryland, 1992.

[Z] M. Zerner, Domaines d'holomorphie des fonctions vérifiant une équation aux dérivées partielles, C. R. Acad. Sci. Paris 272 (1971), 1646-1648. 